08

\title{
Изготовление и исследование зеркал с широкой полосой пропускания для синхротронных применений
}

\author{
(C) А.А. Ахсахалян, ${ }^{1}$ С.А. Гарахин, ${ }^{1,}$ Ф.А. Дарьин, ${ }^{2}$ М.В. Зорина, ${ }^{1}$ В.В. Кривенцов, ${ }^{2}$ Д.Д. Першин, ${ }^{3}$ \\ А.Е. Пестов, ${ }^{1}$ Р.С. Плешков, ${ }^{1}$ В.Н. Полковников, ${ }^{1}$ Я.В. Ракшун, ${ }^{2}$ Н.Н. Салащенко, ${ }^{1}$ С.С. Светохин, ${ }^{2}$ \\ М.В. Свечников, ${ }^{1}$ Д.С. Сороколетов, ${ }^{2}$ В.А. Чернов, ${ }^{2}$ Н.И. Чхало ${ }^{1}$
}

${ }^{1}$ Институт фризики микроструктур РАН, 607680 Нижний Новгород, Россия

${ }^{2}$ Институт ядерной фризики им. Г.И. Будкера СО РАН, 630090 Новосибирск, Россия

${ }^{3}$ Новосибирский государственный университет, 630090 Новосибирск, Россия

ๆ e-mail: GarakhinS@yandex.ru

Поступило в Редакцию 28 апреля 2021 г.

В окончательной редакции 28 апреля 2021 г.

Принято к публикации 28 апреля 2021 г.

Разработаны, изготовлены и проанализированы широкополосные W/Si-многослойные зеркала для широкополосного монохроматора, рассчитанного для спектрального диапазона 7-10 keV. Показана возможность использования стекового подхода для получения многослойных зеркал с коэффициентом отражения около $30 \%$ со спектральной полосой пропускания $\Delta E / E$ около $20 \%$. Приведены результаты измерений угловых и спектральных кривых отражения зеркала, полученных на лабораторном дифрактометре и на экспериментальной станции „Рентгенофлуоресцентного элементного анализа“ накопителя ВЭПП-3 ИЯФ CO PAH.

Ключевые слова: жесткий рентгеновский диапазон, монохроматор, синхротронное излучение, широкополосные зеркала, стековые структуры, многослойные рентгеновские зеркала.

DOI: $10.21883 /$ JTF.2021.10.51366.128-21

\section{Введение}

На протяжении 40 лет периодические многослойные зеркала (ПМЗ) являются универсальным инструментом для построения оптических схем, предназначенных для работы во всем рентгеновском и экстремальном ультрафиолетовом (ЭУФ) диапазонах длин волн $(0.01-60 \mathrm{~nm})$. Уникальность ПМЗ по сравнению с другими рентгенооптическими элементами связана с высокими (до 70\%) коэффициентами отражения при нормальных углах падения в ЭУФ и мягком рентгеновском (MP) диапазонах, что обеспечивает высокую числовую апертуру, минимальные аберрации и эффективное использование излучения источника. Развитие технологи изготовления и методов аттестации таких структур в настоящий момент позволило вплотную подойти к теоретическому пределу значений коэффициентов отражения [1-5]. Спектральная селективность ПМЗ (отношение резонансной длины волны к спектральной ширине на полувысоте коэффициента отражения) составляет 20-500, что позволяет отстроиться от близко лежащих линий и минимизировать фоновые засветки. Однако ПМЗ не позволяют решать задачи, где нужна широкая полоса пропускания $d E / E$. Поэтому практически с самого начала $[6,7]$ стояла проблема изготовления зеркал с изменяемым по глубине периодом, обладающих большим (по сравнению с периодическими зеркалами) интегральным коэффициен- том отражения, уширенной спектральной или угловой полосой отражения. Так, для астрофизического телескопа, построенного по схеме Киркпатрика-Байеза [8], рассматривались $\mathrm{W} / \mathrm{Si}$-многослойные зеркала, работающие на длинах волн до $0.18 \AA$ при скользящих углах падения $3 \mathrm{mrad}$. Период зеркала монотонно уменьшался вглубь структуры по закону $d_{i}=a(b+i)-c$, где $d_{i}-$ величина $i$-го периода от поверхности, $c \approx 0.26, a$ и $b$ представляют собой параметры оптимизации. Этот подход носит название АВС-модели или суперзеркала. В работах $[9,10]$ были предложены численные и аналитические способы расчета параметров таких зеркал, удовлетворяющих требуемому профилю коэффициента отражения. Этот подход был применен при конструировании зеркал для каналов синхротронного излучения и увеличения потока от рентгеновской трубки на образце [11].

Разработке и применению апериодических зеркал в области мягкого рентгеновского и ЭУФ излучения посвящена монография [12].

Несмотря на успехи, достигнутые в области синтеза апериодических зеркал [13-15], на практике эта задача остается довольно сложной. Главные трудности при изготовлении рентгеновского зеркала, состоящего из большого числа (до 100 и более) слоев с индивидуальными толщинами, обладающего требуемым коэффициентом отражения, связаны, прежде всего, со сложностью и 

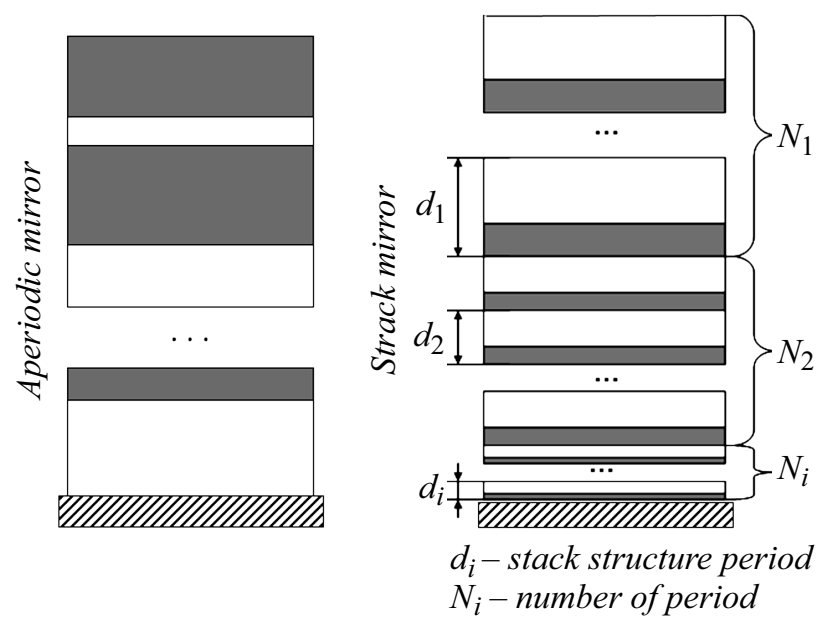

Рис. 1. Апериодические и стековые структуры.

длительностью процесса калибровки. Технологические ошибки в толщинах слоя порядка $1-2 \%$, не сказывающиеся существенно на коэффициент отражения периодической многослойной структуры, в широкополосном зеркале могут вносить заметные искажения [16]. На практике, чтобы с достаточной точностью напылить $N$ различных толщин, необходимо провести порядка $N$ разных калибровок. При этом нужно, чтобы материалы были достаточно хорошо изучены на стадии изготовления периодических зеркал, т.е. известны как плотности пленок, так и межслоевые шероховатости. Такое априорное знание необходимо, поскольку результат решения задачи оптимизации будет существенно определяться шероховатостями, т.е. оптимальные толщины апериодического многослойного зеркала будут разными в зависимости от характеристик границ. Далее после изготовления зеркала проводится его аттестация. В случае отклонения от расчетных параметров необходимо решить задачу реконструкции внутреннего строения зеркала для дальнейшей коррекции технологического процесса. Решение обратной задачи в классе апериодических структур очень сложно, зачастую никакой существенной информации о строении отдельных пленок по данным рефлектометрических измерений получить не удается.

Для некоторого типа задач удается применить подход, предложенный в [17] для рентгеновского телескопа „жесткого“ рентгеновского диапазона $(20-40 \mathrm{keV}$, угол падения излучения $0.3^{\circ}$ ). В работе [17] широкополосное отражающее покрытие состояло из 8 напыленных друг на друга периодических $\mathrm{Pt} / \mathrm{C}$-многослойных зеркал с периодами 60-30 А, убывающими к подложке. Всего структура состояла из 168 слоев, причем толщина $\mathrm{Pt}$ фиксировалась на уровне $15 \AA$, кроме верхнего слоя, в котором доли $\mathrm{Pt}$ и $\mathrm{C}$ выбирались равными с целью подавления второго брэгговского пика. Из-за своей простоты этот метод был успешно применен при разработке рентгеновских зеркал скользящего падения для рентге- новских телескопов InFOC $\mu \mathrm{S}$ и ASTRO-H, а также для других применений [18-20].

Апериодические зеркала такого типа - стековые, состоят из нескольких периодических зеркал со смещенными резонансными пиками отражения (рис. 1). В [21] на примере W/Si-зеркала дается формула для подсчета числа стеков, толщин слоев и их последовательности, которые можно использовать для определения стартовой структуры при оптимизации структуры зеркала.

$\mathrm{B}$ настоящей работе на примере $\mathrm{W} / \mathrm{Si}$-многослойных зеркал для широкополосного монохроматора, предназначенного для спектрального диапазона 7-10 keV, подробно описываются развитые нами методы оптимизации, изготовления и реконструкции внутреннего строения образцов по данным рентгеновского отражения. Приводятся результаты измерений угловых и спектральных кривых отражения зеркала, полученных на лабораторном дифрактометре и экспериментальной станции „Рентгенофлуоресцентного элементного анализа“ накопителя ВЭПП-3 ИЯФ СО РАН.

Такие широкополосные зеркала могут использоваться в рентгеновской спектроскопии поглощения для подавления глитчей, вызванных вкладом высших порядков дифракции кристаллов.

\section{1. Оптимизация параметров стековой структуры}

Математически задача оптимизации представляет собой минимизацию функционала [14]

$$
F=\int\left[R(\lambda)-R^{\text {target }}\right]^{2 m} d \lambda,
$$

где $R^{\text {target }}$ - целевая функция для коэффициента отражения, определяет высоту „плато“. Интеграл вычисляется в области определения $R^{\text {target }}$ и рассматриваемая как функция толщин слоев. Процедура содержит несколько итераций, сначала плато выбирается на уровне, соответствующем периодическому зеркалу, и постепенно понижается до достижения приемлемой гладкости.

Оптимизация производилась при помощи алгоритма дифференциальной эволюции реализованного в рамках программы Multifitting, разработанной в [22]. В отличие от широко распространенного аналога IMD [23]

Таблица 1. Расчетные толщины слоев Mo/Si стековых многослойных зеркал для диапазона $7-10 \mathrm{keV}$

\begin{tabular}{c|c|c|c}
\hline $\begin{array}{c}\text { Номер ПМ3 от } \\
\text { поверхности }\end{array}$ & $\begin{array}{c}\text { Число } \\
\text { периодов, Ni }\end{array}$ & Материал & $\begin{array}{c}\text { Толщины } \\
\text { слоев ПМ3, nm }\end{array}$ \\
\hline 1 & 13 & $\mathrm{Si}$ & 3.89 \\
& 32 & $\mathrm{Mo}$ & 1.92 \\
\hline 2 & & $\mathrm{Si}$ & 2.66 \\
& & $\mathrm{Mo}$ & 2.82
\end{tabular}


Multifitting позволяет рассматривать число периодов в стеках Ni как параметр, что делает возможным решение задачи оптимизации в классе стековых структур. Кроме того, переходные слои представляются в виде линейной комбинации простейших функций, включая функцию ошибок, наилучшим образом описывающую шероховатость и step-функцию, описывающую стехиометрические слои в переходной области [24].

В случае стекового многослойного зеркала число восстанавливаемых параметров существенно меньше, обратная задача может быть решена с высокой степенью точности, и проведена соответствующая коррекция технологического процесса. Приведенные соображения объясняют преимущество использования стекового дизайна при изготовлении широкополосных рентгеновских зеркал там, где это возможно.

Для расчета широкополосного зеркала, обеспечивающего в диапазоне 7-10 keV равномерное отражение с коэффициентом $R \geq 30 \%$, спектральную полосу отражения $\Delta E / E \sim 20 \%$ и подавление 3-й гармоники не менее чем $10^{3}$, были рассмотрены структуры $\mathrm{Mo} / \mathrm{Si}$ и $\mathrm{W} / \mathrm{Si}$. При вычислениях закладывались ширины переходных
Таблица 2. Расчетные толщины слоев W/Si стековых многослойных зеркал для диапазона $7-10 \mathrm{keV}$

\begin{tabular}{c|c|c|c}
\hline $\begin{array}{c}\text { Номер ПМ3 от } \\
\text { поверхности }\end{array}$ & $\begin{array}{c}\text { Число } \\
\text { периодов, } N_{i}\end{array}$ & Материал & $\begin{array}{c}\text { Толщины } \\
\text { слоев ПМ3, nm }\end{array}$ \\
\hline 1 & 6 & $\mathrm{Si}$ & 2.78 \\
& & $\mathrm{~W}$ & 2.23 \\
\hline 2 & 9 & $\mathrm{Si}$ & 1.40 \\
& 21 & $\mathrm{~W}$ & 3.05 \\
\hline 3 & & $\mathrm{Si}$ & 1.43 \\
& & $\mathrm{~W}$ & 2.97
\end{tabular}

областей Mo-на-Si, равные $1.2 \mathrm{~nm}$, и $\mathrm{Si}-$ на-Mo - $0.6 \mathrm{~nm}$, характерные для Mo/Si-многослойных зеркал [25-27], плотности материалов пленок табличные. Для структуры W/Si ширины переходных областей были $0.3 \mathrm{~nm}$ на обеих границах [28]. Состав оптимизированных структур приведен в табл. 1 и 2.

Из рис. 2 и 3 видно, что Mo/Si-структура лучше по показателю подавления высших гармоник, но хуже по
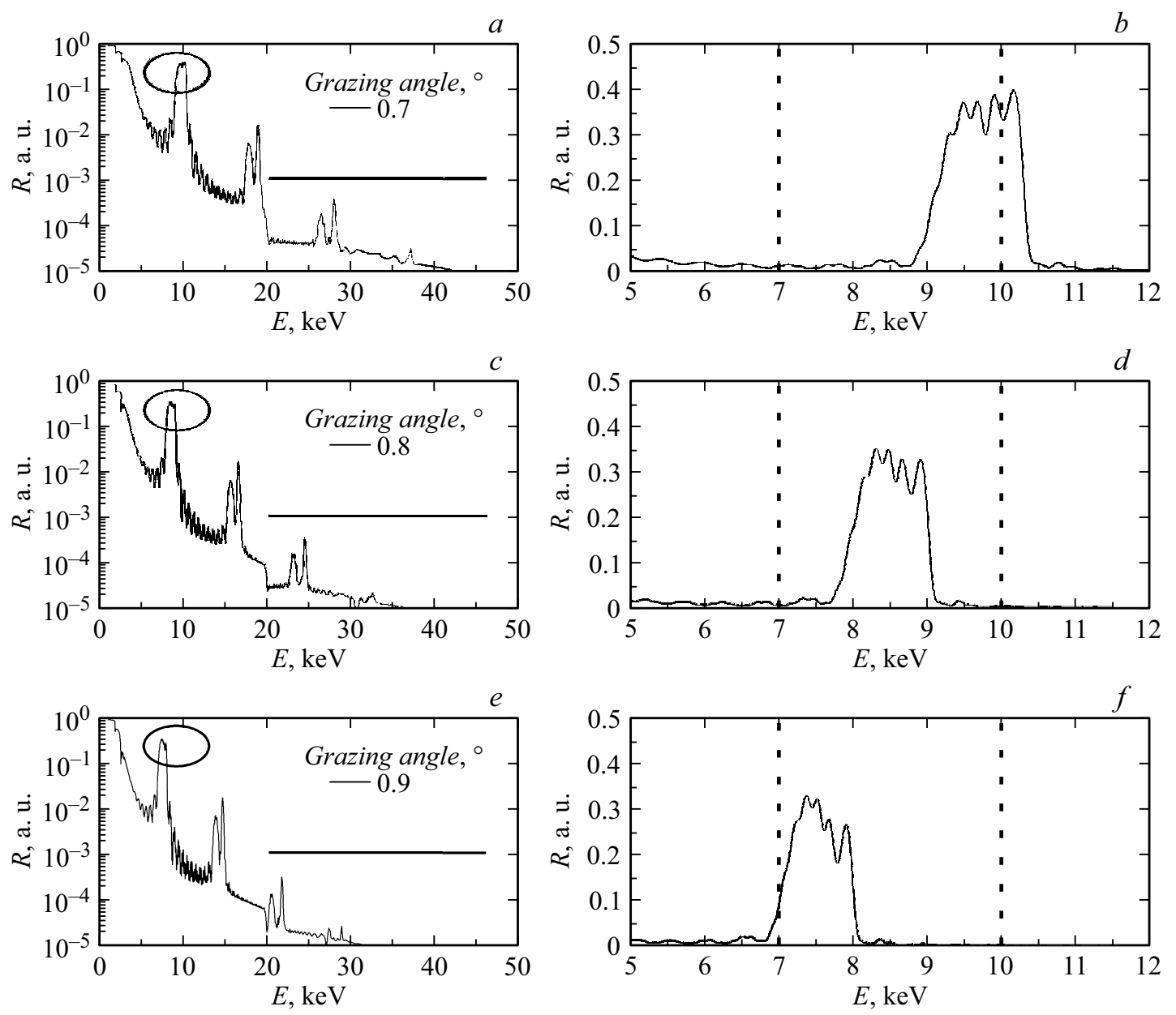

Рис. 2. Зависимости $R(E)$ для структуры $\mathrm{Mo} / \mathrm{Si}$ при угле $0.7^{\circ}(a, b), 0.8^{\circ}(c, d)$ и $0.9^{\circ}(e, f)$ с указанием высших порядков отражения (рисунки слева). Справа (в масштабе) коэффициент отражения в рабочем диапазоне энергий $7-10 \mathrm{keV}$ для соответствующих углов. 

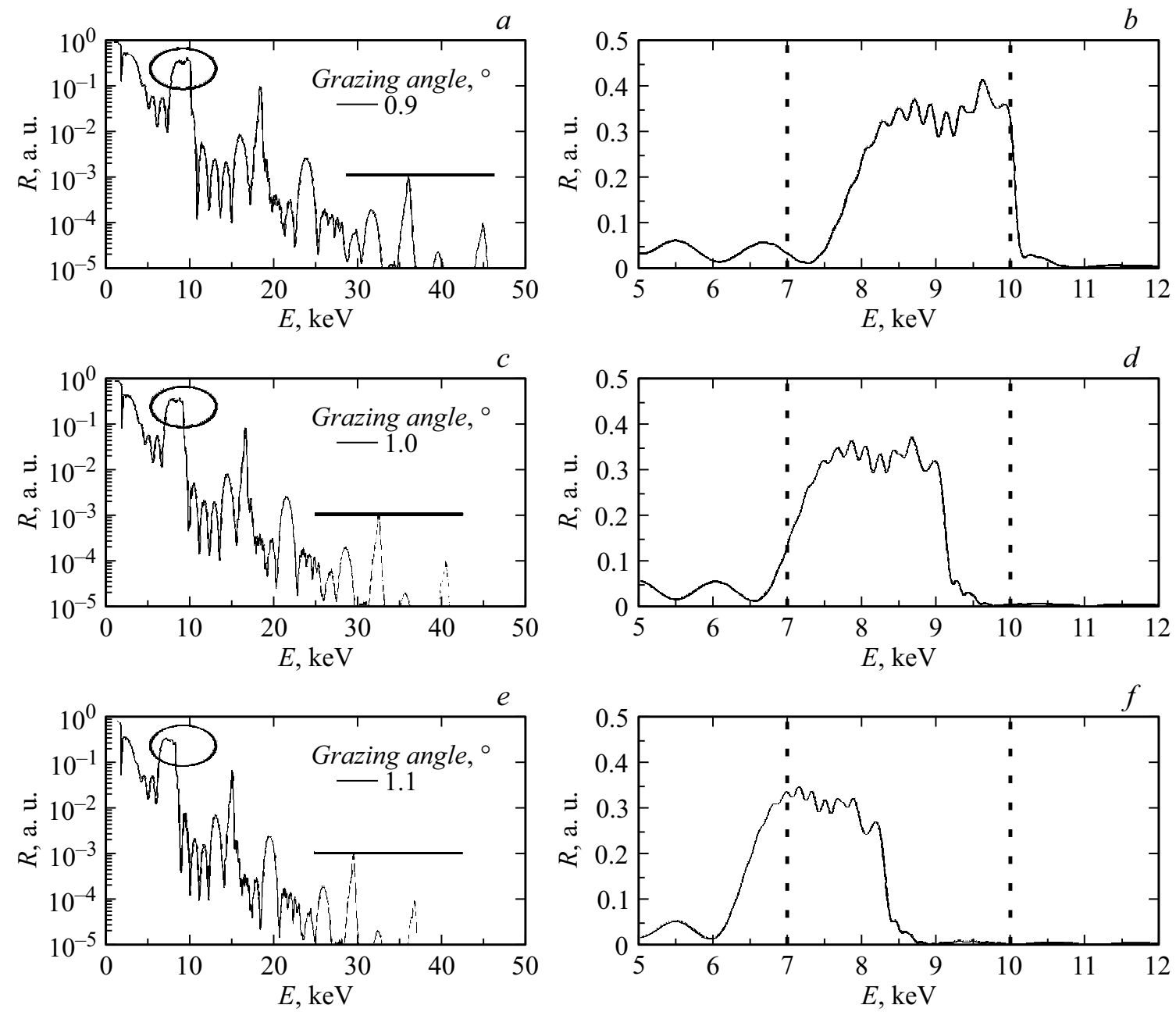

Рис. 3. Рассчитанные зависимости $R(E)$ для стековой структуры $\mathrm{W} / \mathrm{Si}$ при угле $0.7^{\circ}(a, b), 0.8^{\circ}(c, d)$ и $0.9^{\circ}(e, f)$ с указанием высших порядков отражения (рисунки слева). Справа (в масштабе) коэффициент отражения в рабочем диапазоне энергий $7-10 \mathrm{keV}$ для соответствующих углов.

коэффициенту отражения и гладкости, и имеет более узкую полосу отражения при заданном коэффициенте отражения. Также рабочие углы у Mo/Si-зеркал меньше, чем y W/Si. Поэтому для синтеза и исследований была выбрана структура W/Si.

\section{2. Методика эксперимента}

Образцы изготавливались методом магнетронного напыления в атмосфере аргона при давлении $1 \cdot 10^{-3}$ Torr; в качестве подложек использованы полированные кварцевые подложки с эффективной шероховатостью $\sigma=0.2-0.3 \mathrm{~nm}$ в диапазоне пространственных частот 0.024-65 $\mu \mathrm{m}^{-1}$. В качестве подслоя, позволяющего химически стравить многослойное зеркало в случае неудачного процесса напыления, использовалась многослойная структура $\mathrm{Cr} / \mathrm{Y}$. Подробное описание технологического процесса и установки содержится в [29]. Толщина осажденной пленки материала определяется време-

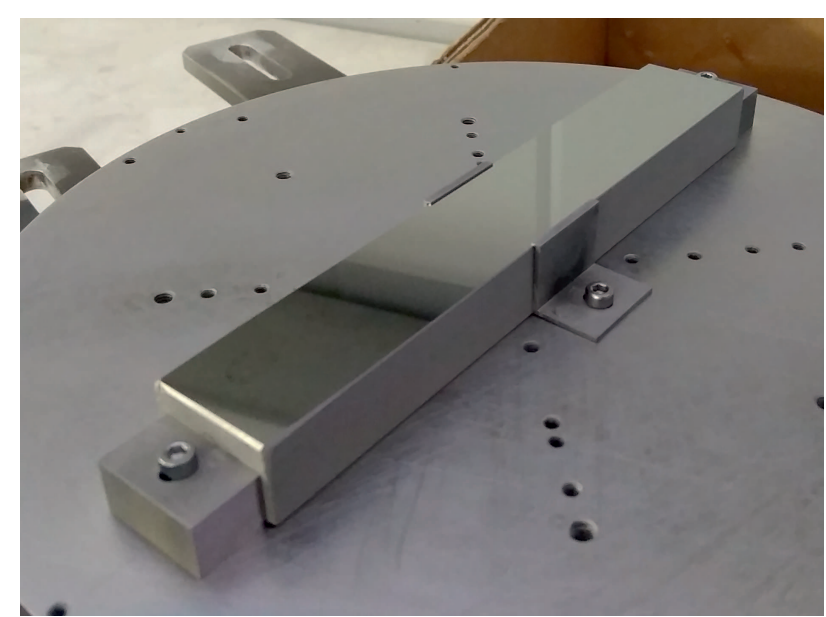

Рис. 4. Фотография синтезированного зеркала в крепеже на напылительном подложкодержателе. 


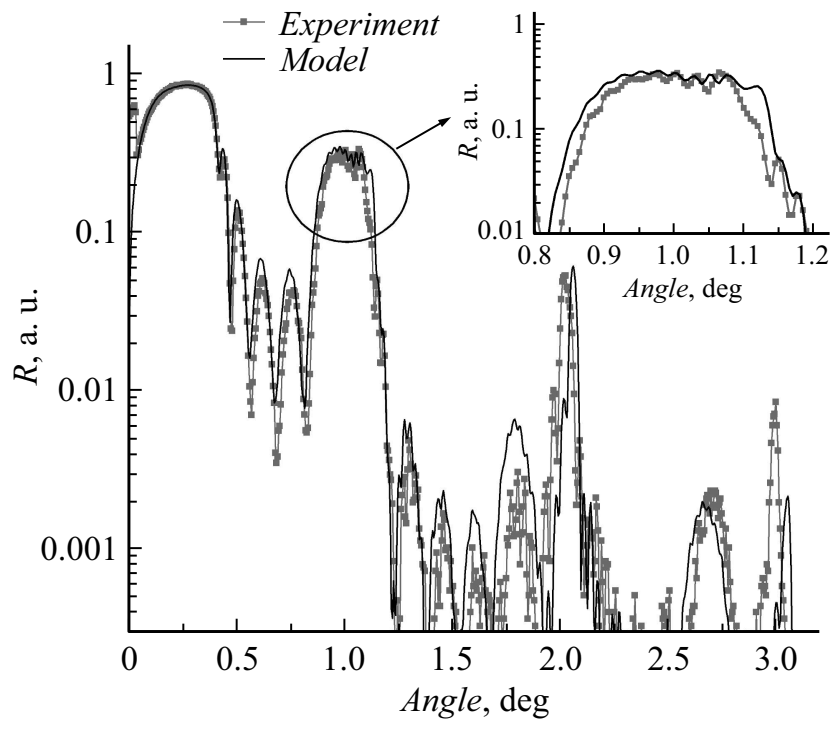

Рис. 5. Измеренная и расчетная угловые зависимости коэффициентов отражения стековой структуры $\mathrm{W} / \mathrm{Si}$ на $\lambda=0.154 \mathrm{~nm}$. Точки - экспериментальные данные, сплошная линия моделирование структуры.

нем прохождения подложки над мишенью и величиной тока разряда, поэтому процессу изготовления зеркала предшествует процедура калибровки, которая состоит в синтезе нескольких предварительных периодических зеркал с толщинами слоев, соответствующими таковым в спроектированной широкополосной структуре.

По данным малоугловой рентгеновской дифракции, полученным с использованием четырехкристального высокоразрешающего дифрактометра PANalitycal X'Pert Pro (длина волны $0.154 \mathrm{~nm}$ ), решается обратная задача, в результате которой с высокой точностью определяются параметры синтеза толщин слоев, содержащихся в требуемом широкополосном зеркале. Подробнее методика реконструкции параметров стека по данным рентгеновского отражения, а также ее применение при решении практических задач описаны в [30,31]. Однако неизбежный дрейф электрических параметров разряда, микропробои, флуктуации и систематические изменения давления рабочего газа могут приводить к отклонению толщин пленок от заданных значений. Процедура изготовления многослойного зеркала - итерационная и требует определения параметров зеркала и соответствующей коррекции процесса синтеза. В качестве параметров оптимизации выступают характеристики стеков, составляющих широкополосное зеркало: толщины и плотности материалов, пленок шероховатости/масштабы переходных областей. В случае получения параметров восстановленной структуры, отличных от заданных, производится коррекция процесса напыления при синтезе следующей структуры.

В настоящей работе было изготовлено два идентичных зеркала. Фотография одного из них на подложкодержателе после нанесения отражающего покрытия приведена на рис. 4. На рис. 5 для одного из образцов приведены измеренная и расчетная угловые зависимости коэффициентов отражения, снятые в широком диапазоне углов, вплоть до 3-го порядка отражения. Справа в верхнем углу подробнее показаны части кривых, соответствующие первому порядку отражения. В целом можно говорить о неплохом совпадении эксперимента и теории.

Также следует отметить хорошую воспроизводимость отражательных характеристик многослойных стековых структур, что иллюстрирует рис. 6, на котором приведены измеренные кривые отражения на длине волны $0.154 \mathrm{~nm}$ для двух последовательно синтезированных зеркал. Из рисунка видно хорошее совпадение кривых.

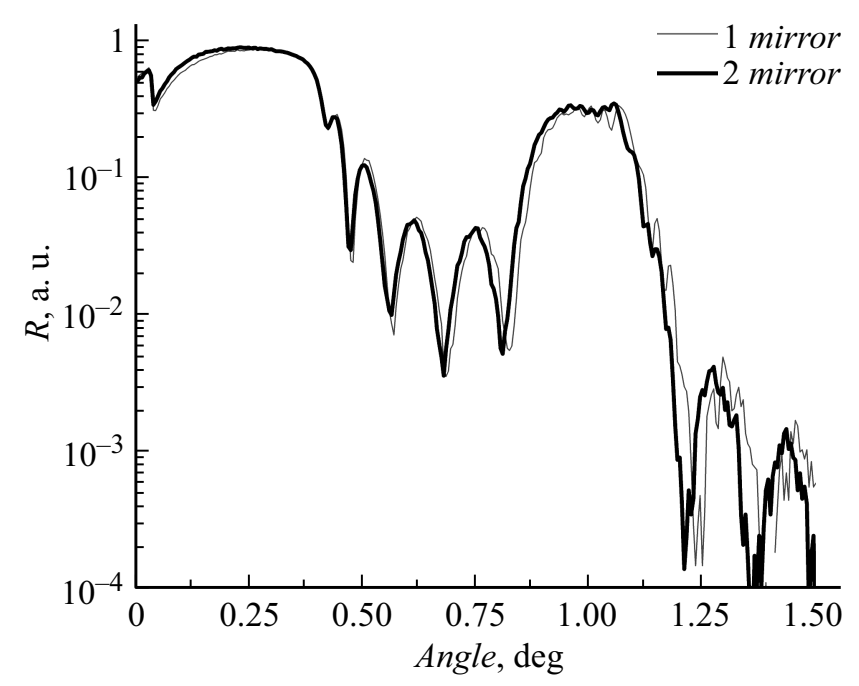

Рис. 6. Сравнение экспериментальных кривых $R(\theta)$ на длине волны $0.154 \mathrm{~nm}$ для двух идентичных синтезированных стековых зеркал.

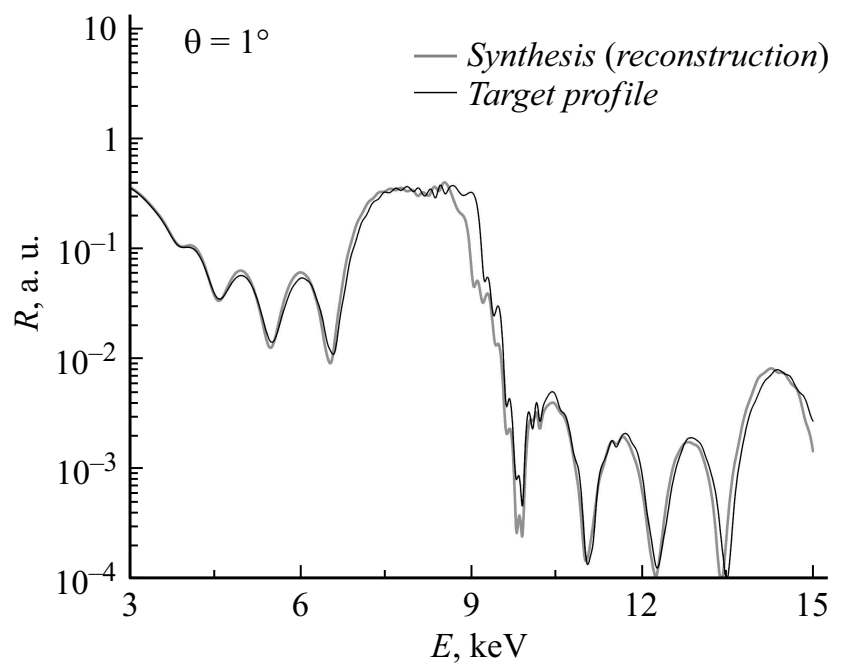

Рис. 7. Результат сравнения ожидаемой (по данным пересчета из угловой зависимости) спектральной кривой отражения с целевым профилем при угле падения излучения на образец $1^{\circ}$. 
В силу отсутствия возможности проведения измерений в целевом спектральном диапазоне, был осуществлен пересчет с использованием параметров стека, полученным из угловой зависимости, снятой на длине волны $0.154 \mathrm{~nm}$, в спектральную при угле падения $1^{\circ}$. На рис. 7 приведены результаты пересчета. Как видно из рисунка, ожидаемая кривая отражения неплохо совпадает с целевой. При этом ширина реального профиля немного уже, чем у целевого, что, по-видимому, связано с некоторыми ошибками толщин пленок при синтезе.

\section{3. Результаты измерений на синхротроне}

Образцы широкополосных зеркал предназначены для спектроскопии рентгеновского поглощения в диапазоне энергий фотонов 7-10 keV. На данном этапе измерения спектральной кривой отражения были проведены на экспериментальной станции „Рентгенофлуоресцентного элементного анализа“ накопителя ВЭПП-3 ИЯФ СО РАН, схема эксперимента представлена на рис. 8.

Измерения проводились в диапазоне энергий $14-25 \mathrm{keV}$, что заметно отличается от расчетного диапазона. Тем не менее эти измерения крайне важны не только для синхротронных применений, но и для технологии и производства широкополосных стековых зеркал. Дело в том, что в лабораторных условиях возможны измерения только угловых зависимостей коэффициентов отражения на узких характеристических линиях материала анода рентгеновской трубки. В нашем случае это линия $\mathrm{Cu} K_{\alpha 1}$. Использование синхротронов на стадии изготовления зеркал существенно увеличит срок изготовления и приведет к значительному росту стоимости таких зеркал. Поэтому крайне важным являлась проверка адекватности метода, основанного на реконструкции параметров стека из угловых зависимостей коэффициентов отражения с

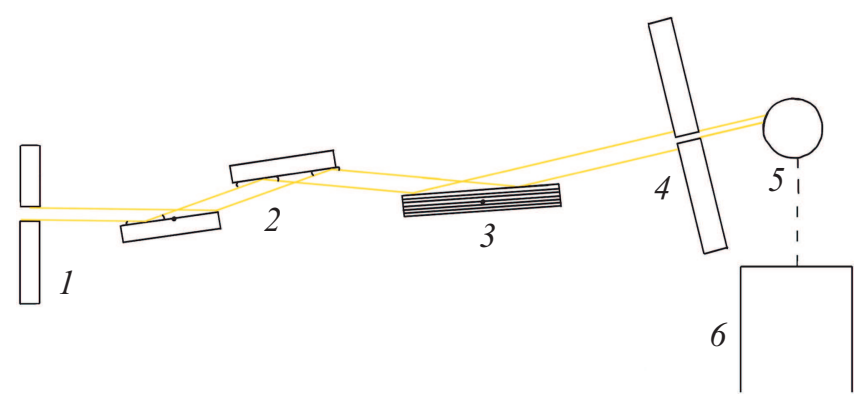

Рис. 8. Схема эксперимента на станции „Рентгенофлуоресцентного элементного анализа“ накопителя ВЭПП-3 ИЯФ СО РАН: 1 - входные щели монохроматора $11 \times 3 \mathrm{~mm}(\amalg \times \mathrm{B})$, 2 - монохроматор-,бабочка “ $\mathrm{Si}(111), 3$ - многослойное зеркало, 4 - выходные щели монохроматора $6 \times 1 \mathrm{~mm}$, для того, чтобы при изменении энергии не менялся сигнал из-за смещения „бабочки“, 5 - рассеиватель (образец стандарта Bil), 6 - детектор $\mathrm{Si}(\mathrm{Li})$.

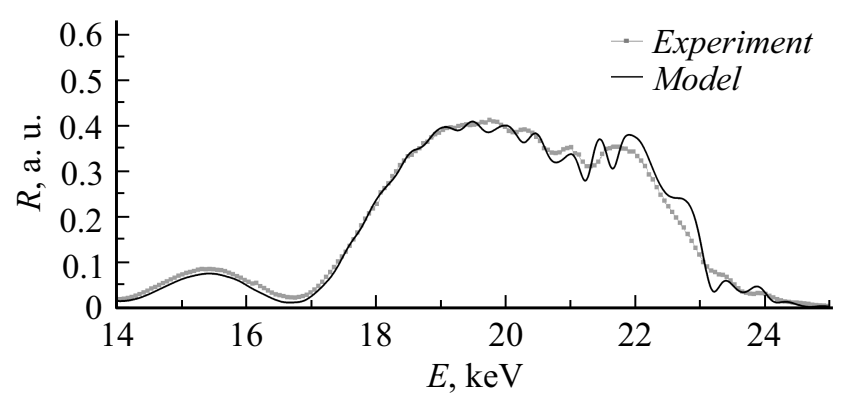

Рис. 9. Измеренная на синхротроне (символы) и рассчитанная (сплошная линия) по восстановленным из кривого отражения на длине волны $0.154 \mathrm{~nm}$ параметрам стека спектральные зависимости коэффициентов отражения.

последующим пересчетом спектральных кривых по этим данным.

На рис. 9 приведена измеренная на синхротроне (символы) и рассчитанная (сплошная линия) по восстановленным из кривой отражения на длине волны $0.154 \mathrm{~nm}$ параметрам стека спектральные зависимости коэффициентов отражения. Как видно из рисунка, наблюдается хорошее соответствие экспериментальной и расчетных кривых, что указывает на адекватность используемого при производстве многослойных стековых зеркал подхода.

\section{Заключение}

Работа посвящена разработке, изготовлению и анализу W/Si-многослойных зеркал для широкополосного монохроматора, рассчитанного для спектрального диапазона 7-10 keV и предназначенного для экспериментов по спектроскопии рентгеновского поглощения. Показана эффективность использования стекового подхода для изготовления и характеризации широкополосных многослойных зеркал для синхротронных применений. Основными результатами данного исследования стало следующее.

Первое, показана эффективность стекового подхода, когда отражающее покрытие состоит из набора (стека) периодических многослойных зеркал с различными резонансными энергиями, для создания широкополосных зеркал для синхротронных применений. Создана пара практически идентичных зеркал, обладающих коэффициентом отражения около $30 \%$ со спектральной полосой пропускания $\Delta E / E$ около $20 \%$ в диапазоне энергий фотонов 7-10 keV.

Второе, несмотря на то, что структура была оптимизирована на конкретный спектральный диапазон, она может использоваться и в других диапазонах без сильной деградации профиля коэффициента отражения.

Третье, показано, что исследование образцов на лабораторном дифрактометре, позволяющем изучать только угловые зависимости коэффициентов отражения на характеристических линиях, является достаточным для 
предсказания спектральных кривых отражения в широком диапазоне энергий фотонов. Последнее крайне важно для технологии изготовления широкополосных многослойных зеркал с заданными спектральными характеристиками.

\section{Финансирование работы}

Работа выполнена при поддержке гранта РФФИ № 20-02-00708 с использованием оборудования ЦКП „Физика и технологии микро- и наноструктур“ при ИФМ PAH.

\section{Конфликт интересов}

Авторы заявляют, что у них нет конфликта интересов.

\section{Список литературы}

[1] N.I. Chkhalo, D.E. Pariev, V.N. Polkovnikov, N.N. Salashchenko, R.A. Shaposhnikov, I.L. Stroulea, M.V. Svechnikov, Yu.A. Vainer, S.Yu. Zuev. Thin Solid Films, 631, 106 (2017). DOI: 10.1016/j.tsf.2017.04.020

[2] N.I. Chkhalo, S.A. Gusev, A.N. Nechay, D.E. Pariev, V.N. Polkovnikov, N.N. Salashchenko, F. Schäfers, M.G. Sertsu, A. Sokolov, M.V. Svechnikov, D.A. Tatarsky. Opt. Lett., 42, 24, 5070 (2017). DOI: 10.1364/OL.42.005070

[3] M.V. Svechnikov, N.I. Chkhalo, S.A. Gusev, A.N. Nechay, D.E. Pariev, A.E. Pestov, V.N. Polkovnikov, D.A. Tatarskiy, N.N. Salashchenko, F. Schafers, M.G. Sertsu, A. Sokolov, Y.A. Vainer, M.V. Zorina. Opt. Express, 26, 26, 33718 (2018). DOI: $10.1364 /$ OE.26.033718

[4] A.E. Yakshin, R.W.E. van de Kruijs, I. Nedelcu, E. Zoethout, E. Louis, F. Bijkerk, H. Enkisch, S. Müllender. Proc. SPIE, 6517, 65170I (2007). DOI: 10.1117/12.711796

[5] S. Bajt. J. Vacuum Sci. Technol. A: Vacuum, Surfaces, and Films, 18 (2), 557 (2000). DOI: 10.1116/1.582224

[6] P. Lee. Opt. Communications, 37 (3), 159 (1981). DOI: $10.1016 / 0030-4018(81) 90408-9$

[7] J.F. Meekins, R.G. Cruddace, H. Gursky. Appl. Opt., 26 (6), 990-4 (1987). DOI: 10.1364/AO.26.000990

[8] K.D. Joensen, P. Voutov, A. Szentgyorgyi, J. Roll, P. Gorenstein, P. Hoghoj, F.E. Christensen. Appl. Opt., 34, 7935 (1995). DOI: 10.1364/AO.34.007935

[9] E. Ziegler, I.N. Bukreeva, I.V. Kozhevnikov, A.S. Pirozhkov, E.N. Ragozin. Proceed. SPIE, 3737, 386 (1999). DOI: $10.1117 / 12.360033$

[10] I.V. Kozhevnikov, I.N. Bukreeva, E. Ziegler. Nucl. Instrum. Method. Phys. Res. A, 460, 424 (2001). DOI: 10.1016/S0168-9002(00)01079-2

[11] C. Morawe, E. Ziegler, J.-C. Peffen, I. Kozhevnikov. Nucl. Instrum. Method. Phys. Res. A, 493 (3), 189 (2002). DOI: $10.1016 / \mathrm{S} 0168-9002(02) 01570-\mathrm{X}$

[12] Е.Н. Рагозин, Е.А. Вишняков, А.О. Колесников, А.С. Пирожков, А.Н. Шатохин. Апериодические элементы в оптике мягкого рентгеновского диапазона (Физматлит, M., 2018)

[13] В.В. Кондратенко, В.Е. Левашов, Ю.П. Першин, А.С. Пирожков, Е.Н. Рагозин. Краткие сообщения по физике ФИАН, 7, 32 (2001).
[14] Е.А. Вишняков, Ф.Ф. Каменец, В.В. Кондратенко, М.С. Лугинин, А.В. Панченко, Ю.П. Першин, А.С. Пирожков, Е.Н. Рагозин. Квант. электрон., 42 (2), 143 (2012). [E.A. Vishnyakov, F.F. Kamenets, V.V. Kondratenko, M.S. Lugin, A.V. Panchenko, Yu.P. Pershin, A.S. Pirozhkov, E.N. Ragozin. Quant. Electron., $42(2), 143$ (2012). DOI: $10.1070 / \mathrm{QE} 2012 \mathrm{v} 042 \mathrm{n} 02 \mathrm{ABEH} 014770]$

[15] Е.А. Вишняков, И.А. Копылец, В.В. Кондратенко, А.О. Колесников, А.С. Пирожков, Е.Н. Рагозин, А.Н. Шатохин. Квант. электрон., 48 (3), 189 (2018). [E.A. Vishnyakov, I.A. Kopylets, V.V. Kondratenko, A.O. Kolesnikov, A.S. Pirozhkov, E.N. Ragozin, A.N. Shatokhin. Quant. Electron., 48(3), 189 (2018). DOI: 10.1070/QEL16574]

[16] П.К. Гайкович, В.Н. Полковников, Н.Н. Салащенко, Н.И. Чхало, Ф. Шаферс, А. Соколов. Квант. электрон., 46 (5), 406 (2016). [P.K. Gaikovich, V.N. Polkovnikov, N.N. Salashchenko, N.I. Chkhalo, F. Schäfers, A. Sokolov. Quant. Electron., 46 (5), 406 (2016). DOI: 10.1070/QEL16037]

[17] K. Yamashita, H. Kunieda, Y. Tawara, K. Tamura, Y. Ogasaka, K. Haga, T. Okajima, Y. Hidaka, S. Ichimaru, S. Takahashi, A. Gotou, H. Kitou, Y. Tsusaka, K. Yokoyama, S. Takeda. Proc. SPIE, 3766, 327 (1999). DOI: 10.1117/12.363646

[18] K. Yamashita, P. J. Serlemitsos, J. Tueller, S.D. Barthelmy, L.M. Bartlett, K. Chan, A. Furuzawa, N. Gehrels, K. Haga, H. Kunieda, P. Kurczynski, G. Lodha, N. Nakajo, N. Nakamura, Y. Namba, Y. Ogasaka, T. Okajima, D. Palmer, A. Parsons, Y. Soong, C.M. Stahl, H. Takata, K. Tamura, Y. Tawara, B.J. Teegarden. Appl. Opt., 37 (34), 8067 (1998). DOI: $10.1364 / A O .37 .008067$

[19] Y. Yao, H. Kunieda, H. Matsumoto, K. Tamura, Y. Miyata. Applied Optics, 52 (27) (2013). DOI: 10.1364/AO.52.006824

[20] K. Tamura, H. Kunieda, Y. Miyata, T. Okajima, T. Miyazawa, A. Furuzawa, H. Awaki, Y. Haba, K. Ishibashi, M. Ishida, Y. Maeda, H. Mori, Y. Tawara, S. Yamauchi, K. Uesugi, Y. Suzuki. J. Astronomi. Telescopes, Instrum., Systems, 4(1), 011209 (2018). DOI: 10.1117/1.JATIS.4.1.011209

[21] X. Wanga. Nucl. Instrum. Method. Phys. Res. Section A, 957 (21), 163435 (2020).

[22] M. Svechnikov. J. Appl. Crystallogr., 53 (1), 244 (2020). DOI: $10.1107 / \mathrm{S} 160057671901584 \mathrm{X}$

[23] D. Windt. Computers in Phys., 12(4), 360 (1998). DOI: $10.1063 / 1.168689$

[24] M. Svechnikov, D. Pariev, A. Nechay, N. Salashchenko, N. Chkhalo, Y. Vainer, D. Gaman. J. Appl. Cryst., 50, 1428 (2017). DOI: 10.1107/S1600576717012286

[25] S. Braun, H. Mai, M. Moss, R. Scholz, A. Lesonet. Jpn. J. Appl. Phys. 41 (6S), 4074 (2002).

DOI: $10.1143 / J J A P .41 .4074$

[26] A.J. Corso, P. Zuppella, P. Nicolosi, D.L. Windt, E.M. Gullikson, M.G. Pelizzo. Opt. Express, 19(15), 13963 (2011). DOI: 10.1364/OE.19.013963

[27] R. Soufli, D.L. Windt, J.C. Robinson, S.L. Baker, E. Spiller, F.J. Dollar, A.L. Aquila, E.M. Gullikson, B. Kjornrattanawanich, J.F. Seely, L Golub. Proc. SPIE, 5901, 59010M (2005). DOI: 10.1117/12.617370

[28] R.V. Medvedev, K.V. Nikolaev, A.A. Zameshin, D.I. Jpes, I.A. Makhotkin, S.N. Yakunin, A.E. Yakshin, F. Bijkerk. J. Appl. Phys., 126, 045302 (2019). DOI: 10.1063/1.5097378 
[29] В.Н. Полковников, Н.Н. Салащенко, М.В. Свечников, Н.И. Чхало. УФН, $190(1), 92-106$ (2020). DOI: $10.3367 /$ UFNr.2019.05.038623 [V.N. Polkovnikov, N.N. Salashchenko, M.V. Svechnikov, N.I. Chkhalo. Phys. Usp., 63, 83 (2020). DOI: 10.3367/UFNe.2019.05.038623]

[30] M.M. Barysheva, S.A. Garakhin, S.Yu. Zuev, V.N. Polkovnikov, N.N. Salashchenko, M.V. Svechnikov, N.I. Chkhalo, S. Yulin. Quant. Electron., 49 (4), 380 (2019). DOI: 10.1070/QEL16990

[31] М.М. Барышева, Е.А. Вишняков, С.А. Гарахин, С.Ю. Зуев, А.С. Кириченко, Кузин, В.Н. Полковников, Н.Н. Салащенко, М.В. Свечников, Н.И. Чхало. ЖТФ, 90 (11), 1876 (2020). DOI: 10.21883/JTF.2020.11.49978.133-20 [S.A. Garakhin, M.M. Barysheva, E.A. Vishnyakov, S.Yu. Zuev, A.S. Kirichenko, S.V. Kuzin, V.N. Polkovnikov, N.N. Salashchenko, M.V. Svechnikov, N.I. Chkhalo. Tech. Phys., 65(11), 1792 (2020). DOI: $10.1134 / \mathrm{S} 1063784220110109]$ 